\title{
Intrathecal versus local infiltration analgesia for pain control in total joint arthroplasty
}

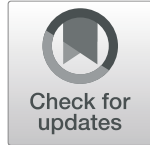

Ai-Lan Cai, Sheng-Jie Liu, Bin Wu and Geng Liu*

\begin{abstract}
Background: The purpose of this meta-analysis was to assess the efficacy of intrathecal morphine (ITM) analgesia and local infiltration analgesia (LIA) for pain control in total joint arthroplasty (TJA).

Methods: Embase, PubMed, the Cochrane Library, and Web of Science were systematically searched for randomized controlled trials (RCTs). All RCTs were comparing intrathecal analgesia and local infiltration analgesia in TJA. Primary outcomes were the visual analog scale (VAS) score with rest or mobilization up to $72 \mathrm{~h}$. Secondary outcomes were the total morphine consumption, length of hospital stay, and morphine-related complications.

Results: Compared with the intrathecal analgesia group, the LIA group was associated with a reduction in VAS score with rest up to $72 \mathrm{~h}$. Moreover, LIA was associated with a decrease in VAS score with mobilization at $6 \mathrm{~h}, 12 \mathrm{~h}$, $48 \mathrm{~h}$, and $72 \mathrm{~h}$. Moreover, LIA significantly reduced total morphine consumption (weighted mean difference (WMD) $=-15.37,95 \% \mathrm{Cl}-22.64$ to $-8.83, P=0.000)$, length of hospital stay $(\mathrm{WMD}=-1.39,95 \% \mathrm{Cl}-1.67$ to $-1.11, P=$ 0.000 ), and morphine-related complications (nausea and pruritus).
\end{abstract}

Conclusions: Local infiltration provided superior analgesia and morphine-sparing effects within the first $72 \mathrm{~h}$ compared with ITM following TJA.

Keywords: Intrathecal analgesia, Local infiltration analgesia, Total joint arthroplasty, Meta-analysis

\section{Introduction}

Total joint arthroplasty (TJA) mainly includes total knee arthroplasty (TKA) and total hip arthroplasty (THA). TJA is considered an effective surgical method for the treatment of end-stage osteoarthritis (OA) [1-3]. However, TJA is associated with severe pain after surgery. Effective pain control is crucial for early ambulation and good functional outcomes [4]. Early ambulation can lead to accelerated rehabilitation and a shortened length of hospital stay, which are the essential elements of a fasttrack recovery program. Moreover, effective pain control after TJA could not only increase patient satisfaction but also decrease the economic costs caused by the length of hospital stay $[5,6]$.

\footnotetext{
* Correspondence: xianchui19840712@126.com

Anesthesiology Department, Liaocheng People's Hospital, No.67 DongChang West Road, Liaocheng 252000, Shandong, China
}

Several methods (femoral nerve block [7], intrathecal morphine (ITM) [8], local infiltration analgesia [9], nonsteroidal anti-inflammatory drugs, oral opiates, and gabapentinoids [10]) have been applied in clinical practice for pain control in hip and knee surgeries. Femoral nerve blocks may increase the occurrence of falls and thus have limited clinical use [11]. Oral opiates and gabapentinoids may be associated with complications such as nausea, vomiting, and somnolence [12]. Local infiltration analgesia (LIA) and intrathecal analgesia are two common analgesia methods for pain control in TJA patients. However, it is still inconclusive as to which is preferable for pain relief in TJA. McCarthy et al. [13] concluded that LIA conferred superior analgesia compared with intrathecal morphine at 24 and $48 \mathrm{~h}$ following TKA. While Rikalainen-Salmi et al. reported that LIA might only enable early mobilization after THA, it was not associated with less nausea than ITM. 
Therefore, we performed a meta-analysis of randomized controlled trials (RCTs) to evaluate the efficiency and safety of LIA and ITM for pain control in TJA.

\section{Methods}

This meta-analysis was based on the recommendations of the Cochrane Handbook for Systematic Reviews of Interventions and was written in accordance with the PRISMA checklist (Preferred Reporting Items for Systematic Reviews and Meta-Analyses).

\section{Literature search}

The following electronic databases were independently and extensively searched by two investigators from their inception through April 2019: Embase, PubMed, the Cochrane Library, and Web of Science. The search keywords were centered on the terms "local infiltration analgesia," "intrathecal analgesia," "total knee arthroplasty," and "total hip arthroplasty," which were adjusted to each database as necessary. In addition, the bibliographies of the included studies and dissertations were searched for additional publications. The search language was restricted to English. As all analyses were on previously published studies, ethical approval was not necessary.

\section{Inclusion and exclusion criteria}

The PRISMA guidelines were followed for the inclusion of studies in the meta-analysis. The detailed description of the inclusion criteria is as follows: (1) trials had to be properly randomized; (2) no additional agents or interventions confounded the comparison; (3) the patients in the trials were given a bolus dose via local injection; and (4) with respect to trials with several intervention groups, the eligibility of each individual group was evaluated, and only those qualified were included. Early studies published as a series of articles from the same institution or author that contained significant overlapping data were excluded for fear of multiple publication bias. Additionally, case reports, editorials, experimental studies, conference articles, commentaries, and other studies that failed to provide detailed results were excluded.

\section{Data collection}

After duplicates were removed and the study selection process was completed, the titles and abstracts were

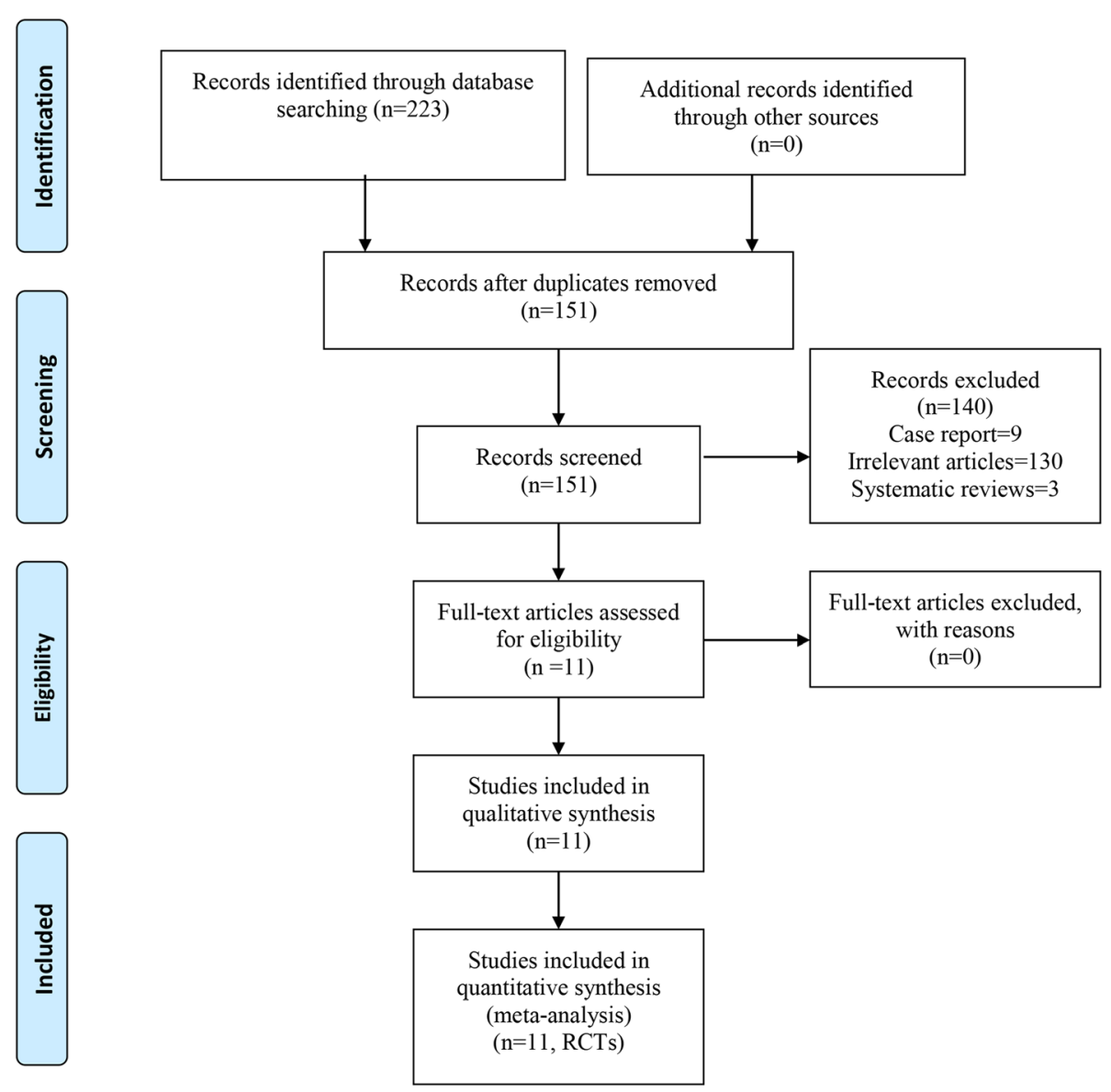

Fig. 1 Flow of trials through the meta-analysis 


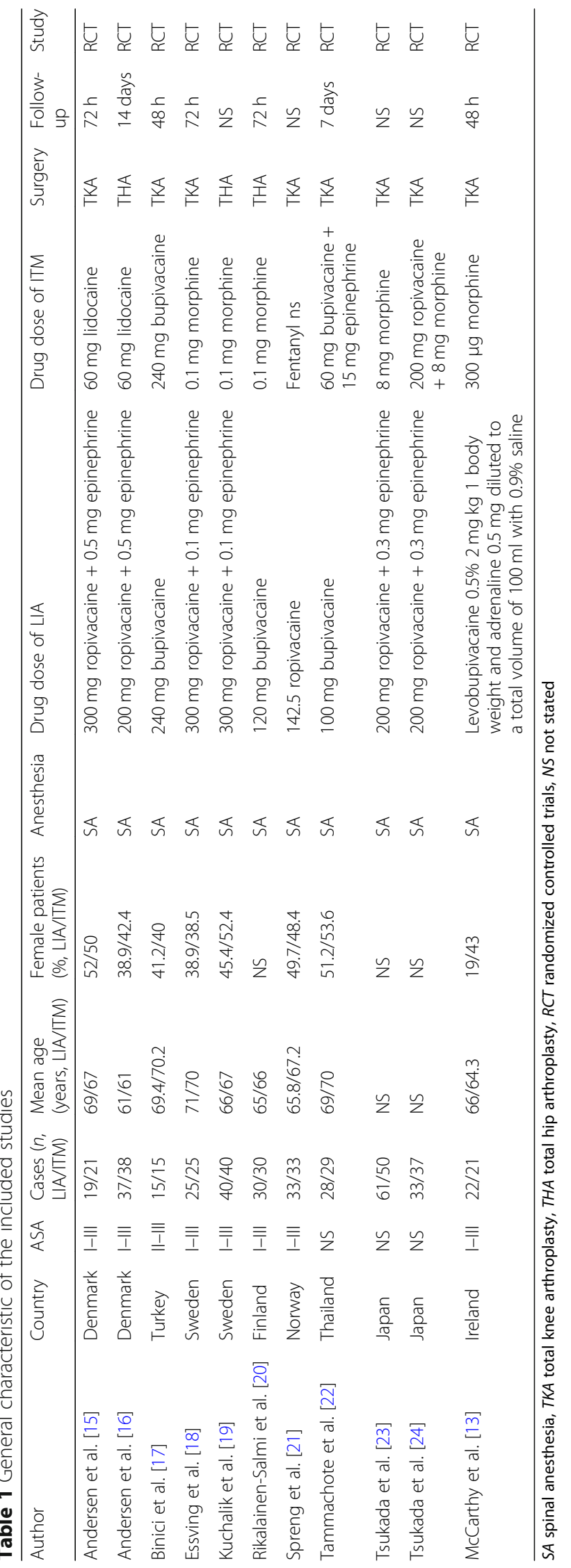


scanned by two independent investigators. The relevant data were extracted by adopting a predetermined standardized procedure that involved the first authors, year of publication, country, ASA, demographic characteristics of the participants (number of cases, mean age, number of female patients), drug dose of LIA, drug dose of ITM, surgery type, follow-up length, and study type. We attempted to contact the study authors for supplementary information when there were insufficient or missing data in the articles.

\section{Quality assessment}

Two reviewers independently assessed the risk of bias in the RCTs using the Cochrane Collaboration's tool [14]. The following items were assessed: (i) random sequence generation (selection bias), (ii) allocation concealment (selection bias), (iii) blinding of participants and personnel (performance bias), (iv) blinding of outcome assessment (detection bias), (v) incomplete outcome data (attrition bias), (vi) selective outcome reporting (reporting bias), and (vii) other bias (other bias). Each item was qualified as low risk $(\mathrm{L})$, unclear risk $(\mathrm{U})$, or high risk $(\mathrm{H})$.

\section{Outcomes}

Pain was assessed using the visual analog scale (VAS) pain score (range, 0 [no pain] to 100 [agonizing pain]). A VAS is a measurement instrument used to quantify the amount of pain reported by the patient. Scores can range from 0 (no pain) to 100 (severest pain). We collected VAS scores with rest or mobilization at $6 \mathrm{~h}, 12 \mathrm{~h}$, $24 \mathrm{~h}, 48 \mathrm{~h}$, and $72 \mathrm{~h}$, total morphine consumption, length of hospital stay, and the occurrence of nausea, pruritus, and respiratory depression in Microsoft ${ }^{\circ}$ Excel (Microsoft Corporation, Redmond, WA, USA).

\section{Statistical analysis}

Stata 12.0 (Stata Corp., College Station, TX) was used to perform the meta-analyses. The overall effect size of each anesthetic was calculated as the weighted average of the inverse variance for study-specific estimates. For dichotomous variables, we listed individual and pooled statistics as odds ratios with 95\% confidence intervals. For continuous data such as the VAS scores with rest or mobilization at $6 \mathrm{~h}, 12 \mathrm{~h}, 24 \mathrm{~h}, 48 \mathrm{~h}$, and $72 \mathrm{~h}$, total morphine consumption, and length of hospital, we pooled the weighted mean time to union with associated 95\% confidence intervals and listed the individual means and standard deviations. Heterogeneity among the individual studies was evaluated based on Cochrane's $Q$ test and the $I^{2}$ index, which express, as a percentage, the proportion of variability in the results due to heterogeneity as opposed to sampling error. Considerable heterogeneity was determined when Cochrane's $Q$ test resulted in
$P<0.10$ and $I^{2}$ greater than $75 \%$. In such cases, a random effect model was selected for analysis. Otherwise, a fixed effect model was used. If needed, a subgroup analysis was conducted to identify and explain the heterogeneity. A $P$ value less than 0.05 was considered significant for all statistical tests.

\section{Results}

\section{Search results}

Figure 1 contains a flowchart that describes the process by which we screened and selected trials. The initial literature search yielded 223 articles in all. In addition, a manual search of relevant references did not identify any additional studies. Duplicate checking and title and abstract screening resulted in 72 publications, and the full texts of all 151 articles were available. Among these, 140

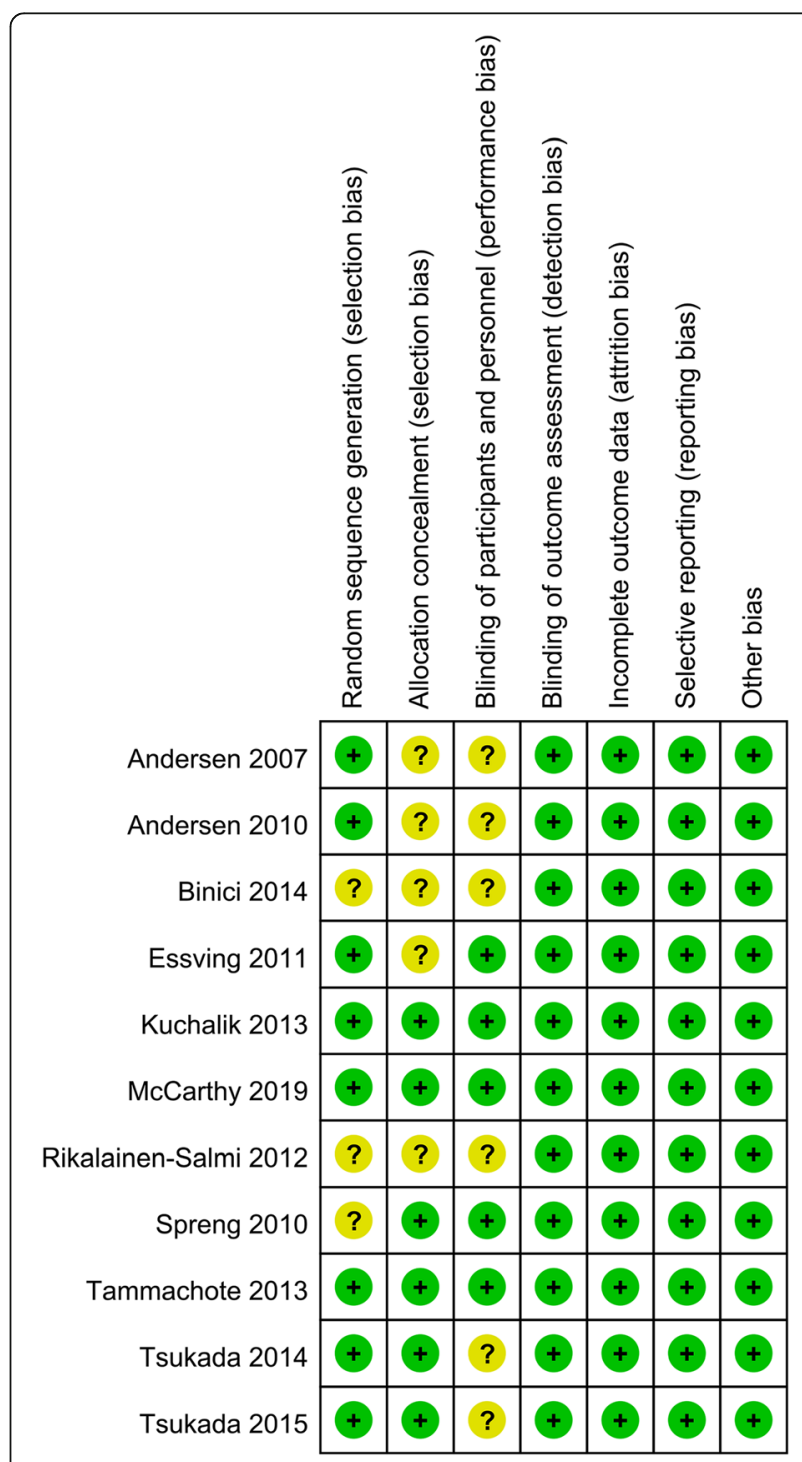

Fig. 2 A, the risk of bias summary, +, no bias; -, bias; ? bias unknown 


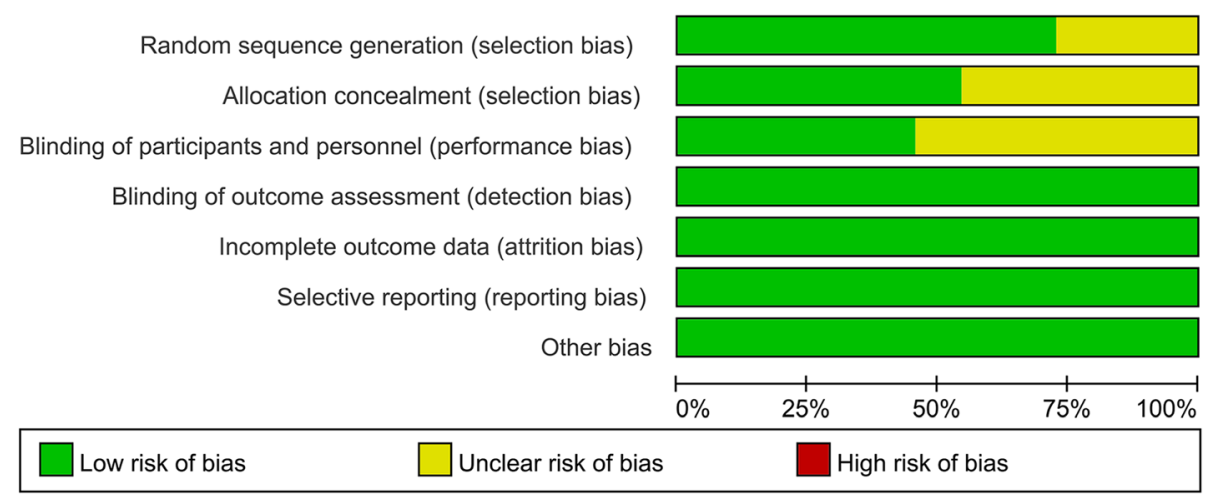

Fig. 3 Risk of bias graph of the included randomized controlled trials

were excluded because they were commentaries, 9 were excluded because they were case reports, 130 were excluded owing to being irrelevant studies, and 3 were excluded because they were systematic reviews. Finally, 11 intermediate- to high-quality studies [13, 15-24] were eligible for inclusion in this meta-analysis.

\section{Characteristics of the trials}

The general characteristics of the included studies are shown in Table 1 . The publication years ranged from 2007 to 2019. The sample size of the included studies ranged from 15 to 61 . The mean age of the included patients ranged from 61 to 71 . Three studies compared intrathecal analgesia with LIA in THA patients, and the remaining studies were in TKA patients. All of the included studies were RCTs. All of the included studies performed spinal anesthesia during surgery.

\section{Risk of bias assessment}

The risk of bias summary and risk of bias graph can be seen in Figs. 2 and 3, respectively. Three studies had an unclear risk of bias for random sequence generation, and five studies had an unclear risk of bias for allocation concealment. Six studies had an unclear risk of bias for blinding of participants and personnel. There was a low risk of bias other bias.

VAS score with rest at $6 \mathrm{~h}, 12 \mathrm{~h}, 24 \mathrm{~h}, 48 \mathrm{~h}$, and $72 \mathrm{~h}$ Eight studies [13, 15, 16, 18-21, 23], with a total of 525 patients, reported the VAS score outcomes $6 \mathrm{~h}$ after TJA. A random-effects model was used because significant heterogeneity was found among the studies $\left(I^{2}=98.8 \%, P=\right.$ 0.000 ). The pooled results demonstrated that the LIA group was associated with a reduction in the VAS score with rest at $6 \mathrm{~h}$ compared with the ITM group (WMD = $-8.23,95 \% \mathrm{CI}-13.87$ to $-2.59, P=0.004$; Fig. 4 ).

Compared with the ITM group, the LIA group was associated with a reduction in VAS score with rest at $12 \mathrm{~h}$ $(\mathrm{WMD}=-10.48,95 \% \mathrm{CI}-16.25$ to $-4.72, P=0.004$; Fig. 4), $24 \mathrm{~h}$ (WMD $=-7.63,95 \% \mathrm{CI}-10.87$ to -4.39 , $P=0.000$; Fig. 4), $48 \mathrm{~h}$ (WMD $=-6.30,95 \% \mathrm{CI}-11.55$ to $-1.05, P=0.019$; Fig. 4 ), and $72 \mathrm{~h}$ (WMD $=-5.97$, $95 \%$ CI -9.09 to $-2.86, P=0.000$; Fig. 4 ).

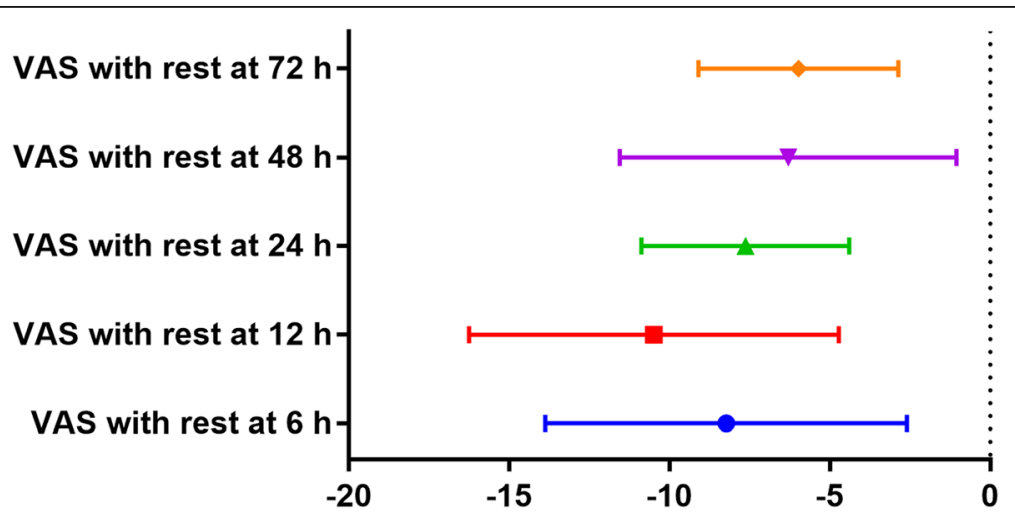

Fig. 4 Forest plots of the included studies comparing the VAS scores with rest at $6 \mathrm{~h}, 12 \mathrm{~h}, 24 \mathrm{~h}, 48 \mathrm{~h}$, and $72 \mathrm{~h}$ 


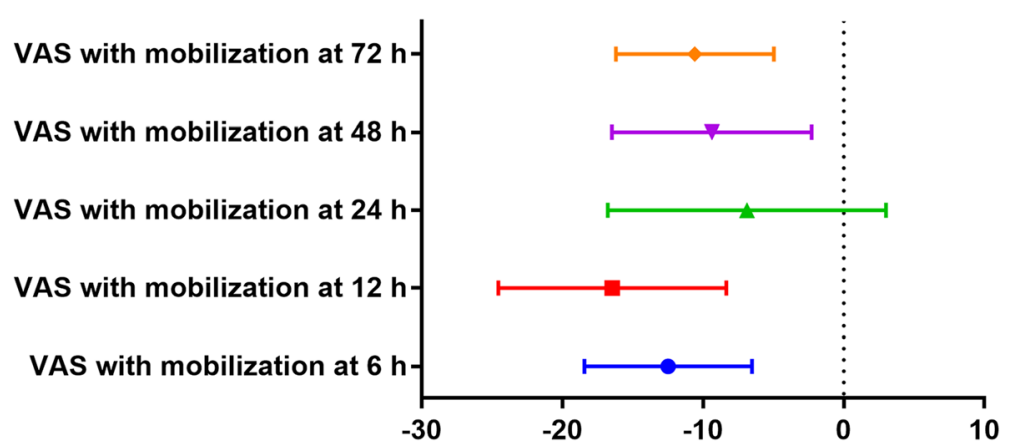

Fig. 5 Forest plots of the included studies comparing the VAS scores with mobilization at $6 \mathrm{~h}, 12 \mathrm{~h}, 24 \mathrm{~h}, 48 \mathrm{~h}$, and $72 \mathrm{~h}$

VAS score with mobilization at $6 \mathrm{~h}, 12 \mathrm{~h}, 24 \mathrm{~h}, 48 \mathrm{~h}$, and $72 \mathrm{~h}$

Compared with the ITM group, the LIA group was associated with a reduction in VAS score with mobilization at 6 h $(\mathrm{WMD}=-12.48,95 \% \mathrm{CI}-18.44$ to $-6.52, P=0.000$; Fig. 5), $12 \mathrm{~h}$ (WMD $=-16.45,95 \% \mathrm{CI}-24.54$ to -8.35 , $P=0.000$; Fig. 5), 24h (WMD $=-6.88,95 \% \mathrm{CI}-16.76$ to $3.00, P=0.172$; Fig. 5 ), $48 \mathrm{~h}$ (WMD $=-9.37,95 \% \mathrm{CI}$ -16.47 to $-2.27, P=0.010$; Fig. 5), and $72 \mathrm{~h}$ (WMD = 10.58, 95\% CI -16.19 to $-4.96, P=0.000$; Fig. 5 ).

\section{Total morphine consumption}

A total of 7 studies with 439 participants reported the total morphine consumption. Compared with the ITM group, the LIA group was associated with a reduction in total morphine consumption (WMD $=-15.37,95 \% \mathrm{CI}$ -22.64 to $-8.83, P=0.000$; Fig. 6 ).

\section{Length of hospital stay}

A total of 7 studies with 332 participants reported the length of hospital stay. Compared with the ITM group,

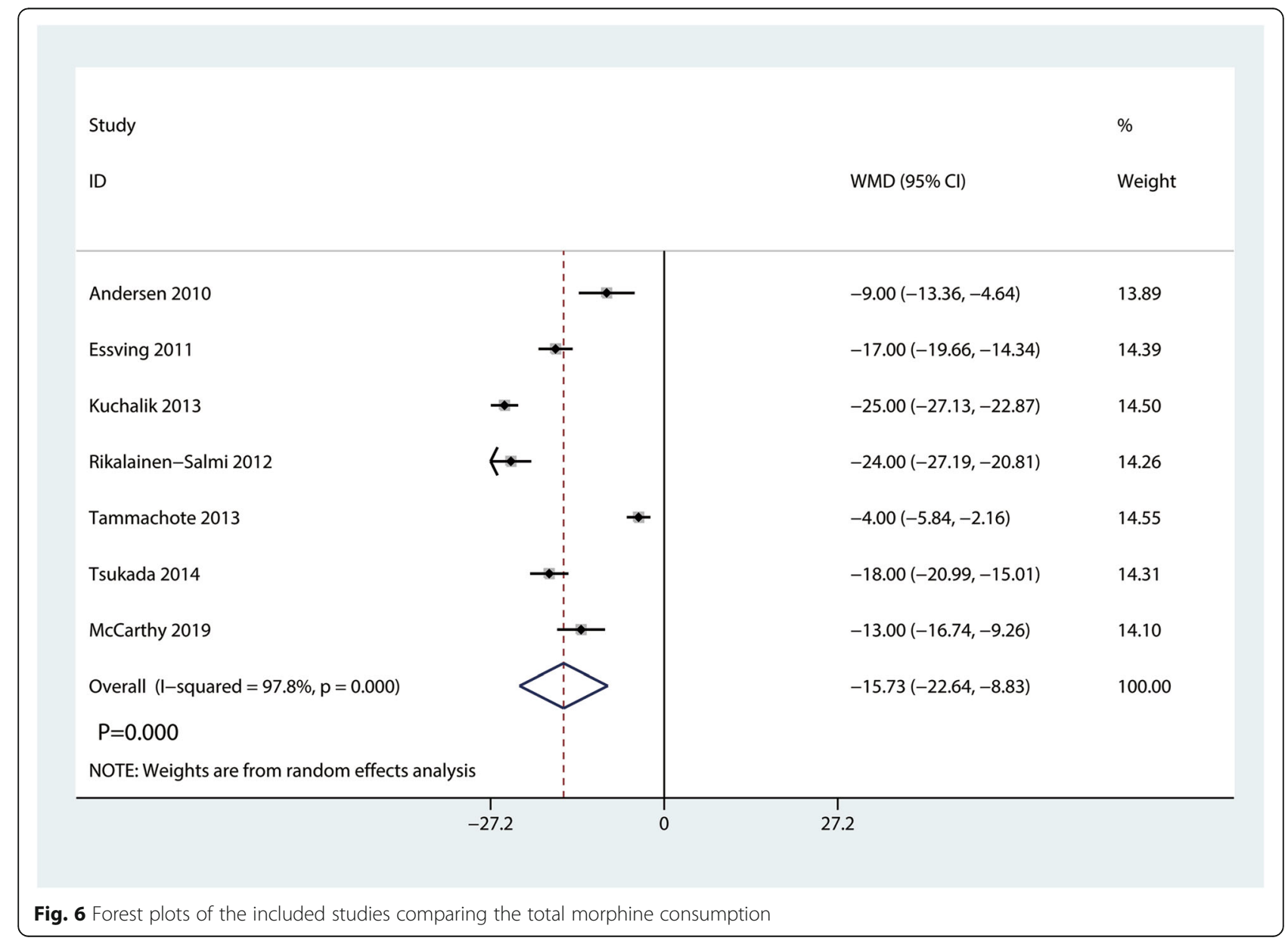


the LIA group was associated with a reduction in the length of hospital stay (WMD $=-1.39,95 \% \mathrm{CI}-1.67$ to $-1.11, P=0.000$; Fig. 7 ).

\section{Occurrence of nausea}

A total of 8 studies with 469 participants reported the occurrence of nausea. Compared with the ITM group, the LIA group was associated with a reduction in the occurrence of nausea $(\mathrm{RR}=0.45,95 \% \mathrm{CI} 0.33$ to $0.60, P=0.000$; Fig. 8$)$.

\section{Occurrence of pruritus}

A total of 7 RCTs with 530 participants reported the occurrence of pruritus. Compared with the ITM group, the LIA group was associated with a reduction in the occurrence of pruritus $(\mathrm{RR}=0.30,95 \% \mathrm{CI} 0.19$ to $0.47, P=0.000$; Fig. 9).

\section{Occurrence of respiratory depression}

A total of 7 RCTs with 460 participants reported the occurrence of respiratory depression. There was no significant difference between the LIA and ITM groups in terms of the occurrence of respiratory depression $(\mathrm{RR}=$ $0.73,95 \%$ CI 0.47 to $1.13, P=0.162$; Fig. 10 ).

\section{Discussion}

\section{Main findings}

Our meta-analysis found that (1) compared with ITM, LIA significantly reduced pain scores with rest or mobilization at $6 \mathrm{~h}, 12 \mathrm{~h}, 24 \mathrm{~h}, 48 \mathrm{~h}$, and $72 \mathrm{~h}$ and (2) LIA further reduced the total morphine consumption, length of hospital stay, and morphine-related complications.

\section{Comparison with other meta-analyses}

Only one relevant meta-analysis has been published [25]. Differences between our meta-analysis and the previous one should be noted. First, the previous meta-analysis included only four trials and 242 patients. In comparison, our current meta-analysis included 11 trials with 382 patients. With the added statistical power of at least 440 cases, our current meta-analysis is the latest and most comprehensive meta-analysis and generally concurs and further reinforces the results from previous meta-analyses. Second, we performed a subgroup analysis and sensitivity analysis. The results from these analyses further confirm our conclusions. Third, we also

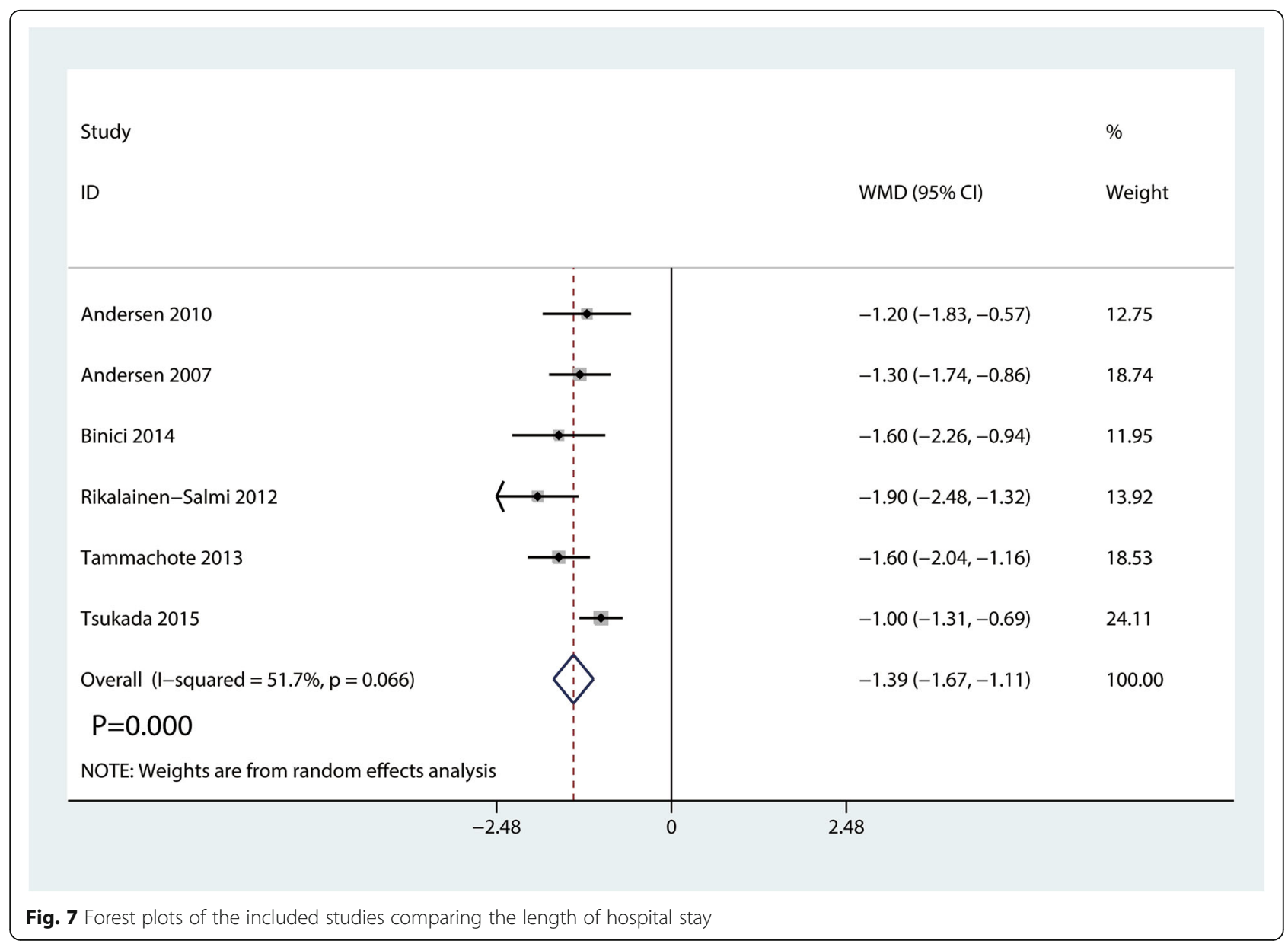




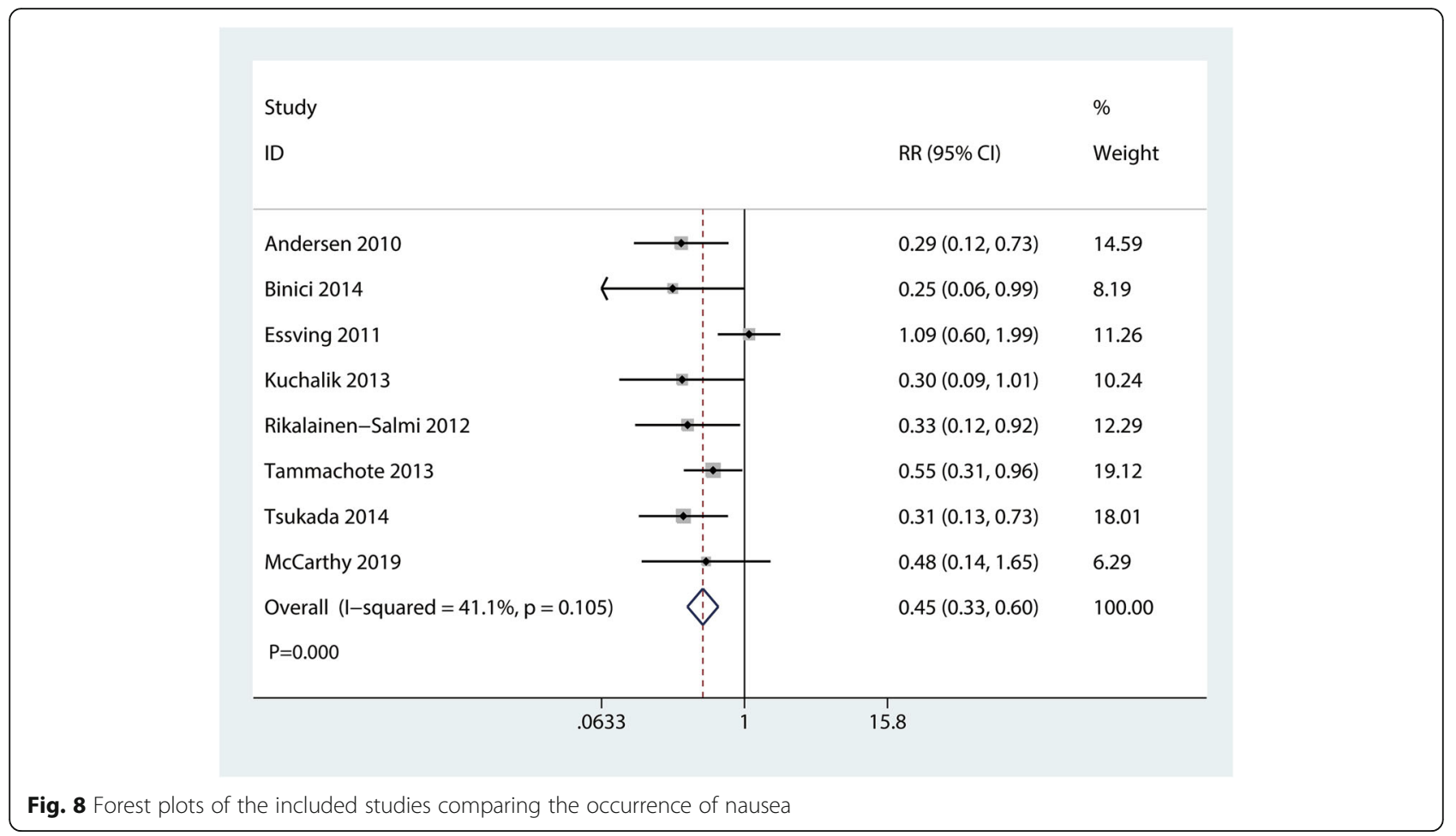

evaluated the effect of LIA and ITM on the length of hospital stay, which is an important index in clinical practice.

This meta-analysis demonstrated that LIA conferred better analgesia with rest up to $72 \mathrm{~h}$. Moreover, LIA was associated with a reduction in morphine consumption compared with the ITM group. Yin et al. [26] conducted a meta-analysis and found that LIA can be used for patients undergoing THA because of its ability to reduce pain scores and analgesic consumption without any additional adverse events.

The results found that LIA could significantly reduce pain scores with mobilization up to $72 \mathrm{~h}$. Pain with mobilization is more important than pain with rest. LIA is not only effective for pain control but also facilitates patients mobilizing early and returning to normal physiological functions quickly. Jia et al. [25] conducted a metaanalysis of LIA and ITM for total knee and hip arthroplasty. In this meta-analysis, LIA was only superior to ITM for pain control within the first $24 \mathrm{~h}$. A major shortcoming of their meta-analysis was that they only included 4 RCTs with hip and knee surgeries. Lalmand et al. [27] revealed that LIA and ITM have equivalent analgesic effects in elective cesarean delivery. Karlsen et al. [28] conducted a review and found that the pain control efficacy of LIA is equivalent to that of other protocols.

Currently, opioids are commonly administered for pain control after TJA. However, morphine was associated with many insupportable complications, such as nausea and vomiting. Thus, total morphine consumption was also measured as the degree of pain control. We found that LIA was associated with a reduction in morphine consumption compared with ITM. These results were in accordance with the reduction in pain.

We measured morphine-related complications (nausea, pruritus, and respiratory depression) between the LIA and ITM groups. The results found that LIA was associated with a reduction in the occurrence of nausea and pruritus. However, there was no significant difference between the LIA and ITM groups in terms of the occurrence of respiratory depression. Kuchálik et al. [29] revealed that LIA was a safe technique for THA during the long-term follow-up (2 years).

There were a total of 5 limitations in the current meta-analysis. (1) Economic costs and functional outcomes for the LIA and ITM groups were not compared due to insufficient data, and future studies should focus on the economic costs and functional outcomes of LIA and ITM. (2) We included TKA and THA patients, and thus, there was high heterogeneity between the included groups. (3) The dose of anesthetics was different in the included studies, and more studies should be focused on the optimal dose and anesthetic drugs for anesthesia. (4) Long-term 


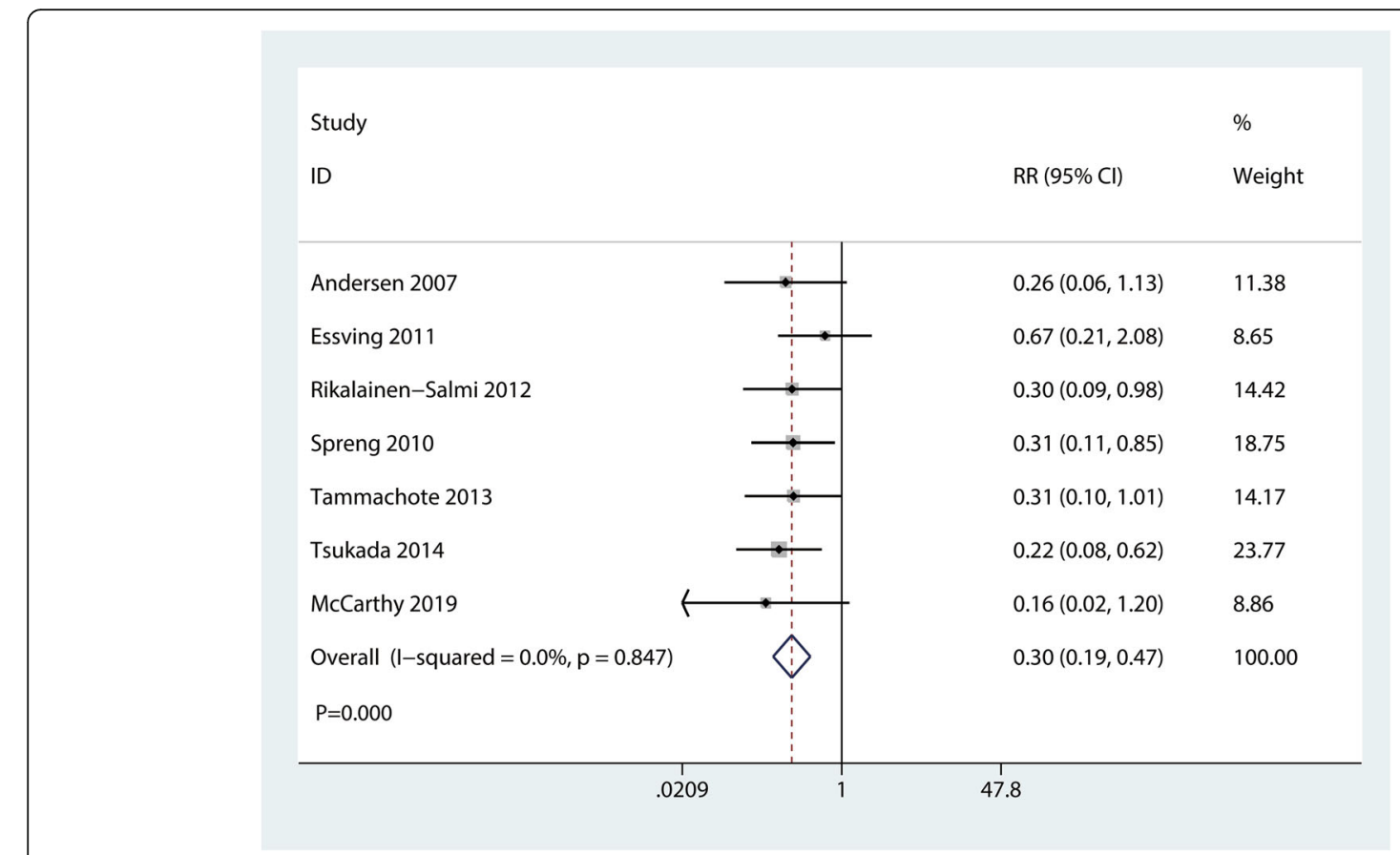

Fig. 9 Forest plots of the included studies comparing the occurrence of pruritus

follow-ups should be performed to reveal the differences in complications of LIA and ITM.

\section{Conclusion}

Local infiltration provided superior analgesia and morphinesparing effects within the first $72 \mathrm{~h}$ compared with ITM following TJA. There were fewer adverse effects in the local infiltration anesthesia groups. However, it should be noted that these conclusions are based on a limited number of studies and patients. Future studies can focus on the economic costs, functional outcomes, and incidence of adverse events to provide more comprehensive results.

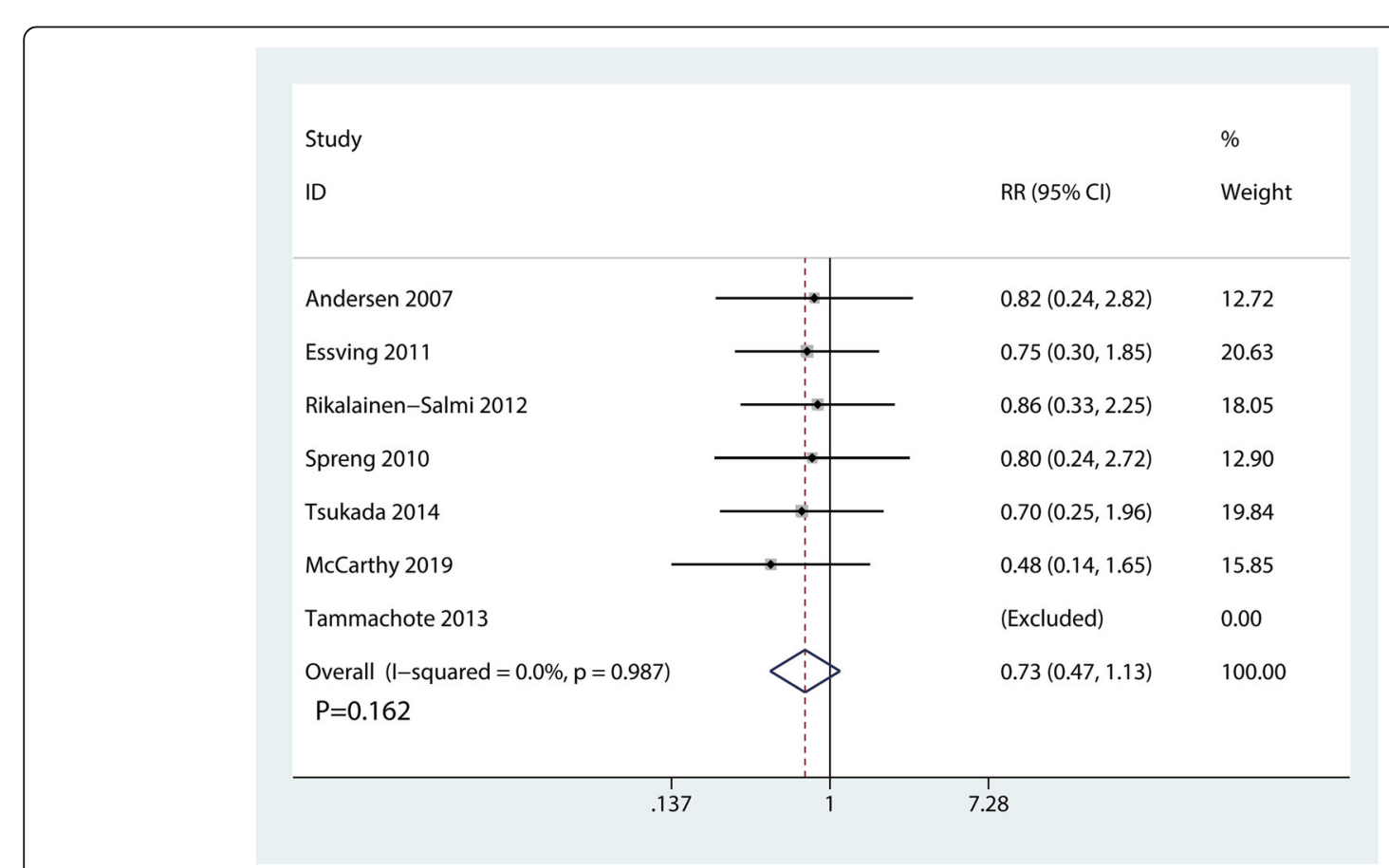

Fig. 10 Forest plots of the included studies comparing the occurrence of respiratory depression 


\section{Abbreviations}

LIA: Local infiltration analgesia; TJA: Total joint arthroplasty;

RCTs: Randomized controlled trials; VAS: Visual analog scale; WMD: Weighted mean difference; TKA: Total knee arthroplasty; THA: Total hip arthroplasty; PRISMA: Preferred Reporting Items for Systematic Reviews and Meta-analyses; L: Low risk; U: Unclear risk; H: High risk; ITM: Intrathecal morphine

\section{Acknowledgements}

None

\section{Authors' contributions}

ALC and SJL conceived the study design. BW performed the study, collected the data, and contributed to the study design. GL and ALC prepared the manuscript. ALC edited the manuscript. The authors read and approved the final manuscript.

\section{Funding}

There is no funding for this article.

\section{Availability of data and materials}

We state that the data will not be shared since all the raw data are present in the figures included in the article.

\section{Ethics approval and consent to participate}

Not applicable

\section{Consent for publication}

Not applicable

\section{Competing interests}

The authors declare that they have no competing interests.

Received: 5 November 2019 Accepted: 9 March 2020

Published online: 18 March 2020

\section{References}

1. Westrich GH, Birch GA, Muskat AR, Padgett DE, Goytizolo EA, Bostrom MP, Mayman DJ, Lin Y, YaDeau JT. Intravenous vs oral acetaminophen as a component of multimodal analgesia after total hip arthroplasty: a randomized, blinded trial. J Arthroplast. 2019.

2. Sun XL, Zhao ZH, Ma JX, Li FB, Li YJ, Meng XM, Ma XL. Continuous local infiltration analgesia for pain control after total knee arthroplasty: a metaanalysis of randomized controlled trials. Medicine. 2015;94(45):e2005.

3. Dong CC, Dong SL, He FC. Comparison of adductor canal block and femoral nerve block for postoperative pain in total knee arthroplasty: a systematic review and meta-analysis. Medicine. 2016;95(12):e2983.

4. Soffin EM, Wu CL. Regional and multimodal analgesia to reduce opioid use after total joint arthroplasty: a narrative review. HSS journal : the musculoskeletal journal of Hospital for Special Surgery. 2019;15(1):57-65.

5. Andersen LO, Kehlet $\mathrm{H}$. Analgesic efficacy of local infiltration analgesia in hip and knee arthroplasty: a systematic review. Br J Anaesth. 2014;113(3): 360-74

6. Affas F. Local infiltration analgesia in knee and hip arthroplasty efficacy and safety. Scand J Pain. 2016:13:59-66.

7. Wang $X$, Sun $Y$, Wang $L$, Hao $X$. Femoral nerve block versus fascia iliaca block for pain control in total knee and hip arthroplasty: a meta-analysis from randomized controlled trials. Medicine. 2017;96(27):e7382.

8. Fournier R, Van Gessel E, Macksay M, Gamulin Z. Onset and offset of intrathecal morphine versus nalbuphine for postoperative pain relief after total hip replacement. Acta Anaesthesiol Scand. 2000;44(8):940-5.

9. Ma LP, Qi YM, Zhao DX. Comparison of local infiltration analgesia and sciatic nerve block for pain control after total knee arthroplasty: a systematic review and meta-analysis. J Orthop Surg Res. 2017;12(1):85

10. Zhai L, Song Z, Liu K. The effect of gabapentin on acute postoperative pain in patients undergoing total knee arthroplasty: a meta-analysis. Medicine. 2016;95(20):e3673.

11. Angers M, Belzile EL, Vachon J, Beauchamp-Chalifour P, Pelet S: Negative influence of femoral nerve block on quadriceps strength recovery following total knee replacement: a prospective randomized trial. Orthopaedics \& traumatology, surgery \& research : OTSR 2019.
12. Klement MR, Bullock WM, Nickel BT, Lampley AJ, Seyler TM, Green CL, Wellman SS, Bolognesi MP, Grant SA: Continuous adductor canal blockade facilitates increased home discharge and decreased opioid consumption after total knee arthroplasty. The Knee 2019.

13. McCarthy D, McNamara J, Galbraith J, Loughnane F, Shorten G, lohom G. A comparison of the analgesic efficacy of local infiltration analgesia vs. intrathecal morphine after total knee replacement: a randomised controlled trial. Eur J Anaesthesiol. 2019;36(4):264-71.

14. Higgins JPT, Green S. Cochrane Handbook for Systematic Reviews of Interventions version 5.1.0 [updated March 2011]. The Cochrane Collaboration, 2011, Chichester, England. Available from www.cochrane-handbook.org ().

15. Andersen KV, Bak M, Christensen BV, Harazuk J, Pedersen NA, Soballe K. A randomized, controlled trial comparing local infiltration analgesia with epidural infusion for total knee arthroplasty. Acta Orthop. 2010;81(5):606-10.

16. Andersen KV, Pfeiffer-Jensen M, Haraldsted V, Soballe K. Reduced hospital stay and narcotic consumption, and improved mobilization with local and intraarticular infiltration after hip arthroplasty: a randomized clinical trial of an intraarticular technique versus epidural infusion in 80 patients. Acta Orthop. 2007;78(2):180-6.

17. Binici Bedir E, Kurtulmus T, Basyigit S, Bakir U, Saglam N, Saka G. A comparison of epidural analgesia and local infiltration analgesia methods in pain control following total knee arthroplasty. Acta Orthop Traumatol Turc. 2014:48(1):73-9.

18. Essving P, Axelsson K, Aberg E, Spannar H, Gupta A, Lundin A. Local infiltration analgesia versus intrathecal morphine for postoperative pain management after total knee arthroplasty: a randomized controlled trial. Anesth Analg. 2011;113(4):926-33.

19. Kuchalik J, Granath B, Ljunggren A, Magnuson A, Lundin A, Gupta A. Postoperative pain relief after total hip arthroplasty: a randomized, doubleblind comparison between intrathecal morphine and local infiltration analgesia. Br J Anaesth. 2013;111(5):793-9.

20. Rikalainen-Salmi R, Forster JG, Makela K, Virolainen P, Leino KA, Pitkanen MT, Neuvonen PJ, Kuusniemi KS. Local infiltration analgesia with levobupivacaine compared with intrathecal morphine in total hip arthroplasty patients. Acta Anaesthesiol Scand. 2012;56(6):695-705.

21. Spreng UJ, Dahl V, Hjall A, Fagerland MW, Raeder J. High-volume local infiltration analgesia combined with intravenous or local ketorolac+ morphine compared with epidural analgesia after total knee arthroplasty. $\mathrm{Br}$ J Anaesth. 2010;105(5):675-82.

22. Tammachote N, Kanitnate S, Manuwong S, Yakumpor T, Panichkul P. Is pain after TKA better with periarticular injection or intrathecal morphine? Clin Orthop Relat Res. 2013:471(6):1992-9.

23. Tsukada S, Wakui M, Hoshino A. Postoperative epidural analgesia compared with intraoperative periarticular injection for pain control following total knee arthroplasty under spinal anesthesia: a randomized controlled trial. J Bone Joint Surg Am. 2014;96(17):1433-8.

24. Tsukada S, Wakui M, Hoshino A. Pain control after simultaneous bilateral total knee arthroplasty: a randomized controlled trial comparing periarticular injection and epidural analgesia. J Bone Joint Surg (Am Vol). 2015;97(5):367-73.

25. Jia XF, Ji Y, Huang GP, Zhou Y, Long M. Comparison of intrathecal and local infiltration analgesia by morphine for pain management in total knee and hip arthroplasty: a meta-analysis of randomized controlled trial. International journal of surgery (London, England). 2017:40:97-108.

26. Yin JB, Cui GB, Mi MS, Du YX, Wu SX, Li YQ, Wang W. Local infiltration analgesia for postoperative pain after hip arthroplasty: a systematic review and meta-analysis. The journal of pain : official journal of the American Pain Society. 2014;15(8):781-99.

27. Lalmand M, Wilwerth M, Fils JF, Van der Linden P. Continuous ropivacaine subfascial wound infusion compared with intrathecal morphine for postcesarean analgesia: a prospective, randomized controlled, double-blind study. Anesth Analg. 2017:125(3):907-12.

28. Karlsen AP, Wetterslev M, Hansen SE, Hansen MS, Mathiesen O, Dahl JB. Postoperative pain treatment after total knee arthroplasty: a systematic review. PLoS One. 2017;12(3):e0173107.

29. Kuchalik J, Magnuson A, Lundin A, Gupta A. Local infiltration analgesia: a 2year follow-up of patients undergoing total hip arthroplasty. Randomized Controlled Trial. 2017;31(6):837-45.

\section{Publisher's Note}

Springer Nature remains neutral with regard to jurisdictional claims in published maps and institutional affiliations. 